\title{
Rupture de la crosse aortique
}

\section{Interrupted Aortic Arch}

\section{A. Millet • J.R. Bazebissa $\cdot$ P.M. Roy}

Reçu le 14 avril 2015 ; accepté le 22 avril 2015

(C) SFMU et Lavoisier SAS 2015

Une patiente de 84 ans est adressée par son centre de dialyse pour une dyspnée fébrile évoluant depuis sept jours. Elle avait été hospitalisée dans un autre hôpital et était sortie avec le diagnostic de pneumopathie gauche. À son arrivée, la pression artérielle est à $110 / 60 \mathrm{mmHg}$, la fréquence cardiaque à $98 \mathrm{bpm}$, la fréquence respiratoire à $22 \mathrm{cpm}$, la $\mathrm{SpO}_{2}$ à $96 \%$ sous $4 \mathrm{~L}$ d'oxygène. À l'examen clinique, on observe une diminution du murmure vésiculaire gauche. La radiographie pulmonaire retrouve un épanchement pleural gauche associé à un élargissement du médiastin. Aucun contexte traumatique ou épisode douloureux n'est mentionné par la patiente. L'angiotomodensitométrie thoracique mettra en évidence une dissection aortique compliquée d'une rupture de la crosse. Un hémothorax gauche et un hémomédiastin sont également visualisés. La patiente refuse toute intervention chirurgicale. Les lésions restent stables au scanner de contrôle à un mois. Elle a pu regagner son EHPAD un mois après la découverte de cette dissection. Un hémothorax gauche peut être révélateur d'une dissection aortique. Lors de la découverte d'un épanchement pleural gauche associé à un élargissement du médiastin, une tomodensitométrie thoracique doit être réalisée à la recherche d'une dissection aortique.

\footnotetext{
A. Millet $(\bowtie) \cdot J . R$. Bazebissa

Département de médecine d'urgence, CHU Angers,

F-49933 Angers cedex 9

e-mail : anthomillet@gmail.com

P.M. Roy

L'UNAM Université, Faculté de médecine, Angers,

EA 3860 CRT « cardioprotection, remodelage et thrombose », département de médecine d'urgence, CHU Angers, F-49933 Angers cedex 9
}

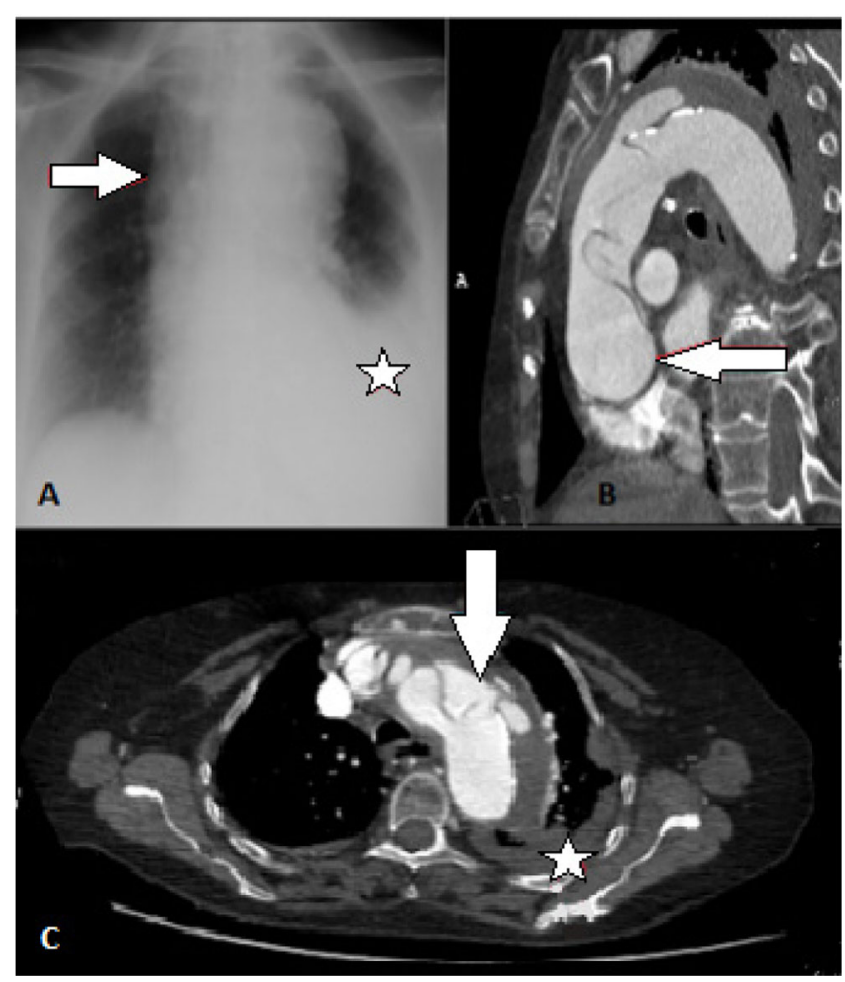

Fig. 1 A : radiographie pulmonaire de face : élargissement du médiastin (flèche), hémothorax (étoile). B : angioscanner thoracique : coupe sagittale, naissance de la dissection aortique au niveau de la valve aortique (flèche). $\mathrm{C}$ : angioscanner thoracique : coupe axiale, hémothorax gauche (étoile), fissuration de la crosse aortique entraînant un hémomédiastin (flèche) 